\title{
Rotating and stationary SPECT system patient motion myocardial perfusion artifacts
}

\author{
Kenneth J. Nichols, PhD, ${ }^{\mathrm{a}, \mathrm{b}}$ and Andrew Van Tosh, $\mathrm{MD}^{\mathrm{c}}$ \\ a Donald and Barbara Zucker School of Medicine at Hofstra/Northwell, Hempstead, NY \\ b Division of Nuclear Medicine and Molecular Imaging, Northwell Health, New Hyde Park, NY \\ c Research Department, St. Francis Hospital, Roslyn, NY
}

Received Feb 26, 2018; accepted Feb 26, 2018

doi: $10.1007 / \mathrm{s} 12350-018-1254-3$

\section{See related article, pp. 1313-1322}

Since the advent of gamma cameras to perform SPECT studies to assess myocardial perfusion, ${ }^{1}$ it has been recognized that patient motion during data acquisition can result in artifacts that can compromise the accuracy of visual and quantitative scan interpretation. ${ }^{2,3}$ Originally, all SPECT systems consisted of one or more Anger detectors that rotated about the patient, so that abrupt patient motion in the caudal direction (corresponding to the " $z$ " direction in the paper by Salvadori et al. ${ }^{4}$ ) produced image data at those projections that are inconsistent with the image counts acquired at other projections. Reconstruction algorithms that are provided with data that are inconsistent from one projection to another often produce artifactual regional decreases in apparent myocardial perfusion that are misinterpreted as genuine perfusion defects. ${ }^{2}$

In recent years, several SPECT systems have become available that are composed of stationary detectors, rather than rotating Anger detectors. ${ }^{5}$ For such systems, all detectors are presented with three-dimensional count distributions that project into multiple detectors in multiple directions simultaneously. So, gamma rays emanating from the heart of a patient who moves caudally will impinge on an entire set of detectors all at the same time. In terms of the image data to be handled by the reconstruction algorithms, this is not unlike PET data. This situation would constitute data

Reprint requests: Kenneth J. Nichols, PhD, Division of Nuclear Medicine and Molecular Imaging, Northwell Health, 270-05 76th Avenue, New Hyde Park, NY 11040; knichols@northwell.edu J Nucl Cardiol 2019;26:1323-6.

1071-3581/\$34.00

Copyright (C) 2018 American Society of Nuclear Cardiology. that at some times are inconsistent with data at other times, but not data at some projections that are inconsistent with data at other projections. Consequently, the same patient executing the same abrupt caudal translation would still cause problems for reconstructing data acquired by a non-rotating system, but just not the same problems as for a rotating system. For a non-rotating SPECT system, a patient with normal myocardial uptake who moved in this way would add anterior wall counts while subtracting inferior wall counts to the reconstructed count volume, so that the entire configuration would be blurred and distorted in the z-direction. Recognizing the inherent differences that the same patient motion would produce in rotating and stationary systems, investigators have developed some approaches to dealing with potential motion problems in data acquired with CZT SPECT systems, specifically. ${ }^{6}$ Overall, one would expect more severe localized artifacts in the case of a rotating SPECT system, compared to which a nonrotating SPECT system would have image data more frequently degraded by image blurring. Thus, the effect of overt patient motion for a non-rotating SPECT system should be similar to the effect of abrupt patient motion on PET scans. Reduced accuracy of myocardial blood flow computations obtained by analyzing cardiac PET data acquired in patients with overt motion problems has been documented, for which optical flow methods have been devised to correct motion and restore myocardial blood flow accuracy. ${ }^{7}$ These correction techniques subsequently evolved to recognize the fact that PET cardiac counts do in fact move as the heart contracts, and to apply those corrections in order to both improve absolute myocardial perfusion quantitation and to quantify the motion of the heart itself in normal sinus rhythm. ${ }^{8}$ Thus, techniques invented to correct for motion problems in PET data evolved to provide the quantified physiologic parameters of regional wall motion and ejection fraction. Even in patients with no overt motion, 
accurate quantitation of perfusion and function from cardiac PET data improves with the application of corrections not only for cardiac contraction but also for respiratory motion, an approach that has received considerable attention. ${ }^{9}$ It can be anticipated that corrections for both types of motion will also benefit the accuracy of parameters quantified from image data acquired by stationary SPECT systems.

In the current issue of the Journal, effects of several types of overt patient motion on reconstructed myocardial counts were simulated for two types of non-rotating SPECT systems, and compared to effects on data acquired by two types of rotating SPECT systems. ${ }^{4}$ Generally speaking, among the various types of potential imaging artifacts, those due to patient motion are particularly troubling because of the wide variability of their effects on scans. Whereas center-of-rotation calibration errors and single detector camera tilt errors produce highly predictable artifactual perfusion pattern problems, ${ }^{10}$ the effects of patient motion are highly variable, since patients can move in numerous ways that include twisting, slumping, sliding, spasming, and coughing motions at different times and at different projection angles during the course of the acquisition. Alterations to baseline myocardial perfusion patterns range from negligible to severe.

While the authors focused their attention on asking whether the non-rotating SPECT systems that they evaluated were less vulnerable to overt patient motion than conventional rotating SPECT systems, a different aspect of the effect of cardiac motion on quantified parameters is to ask whether the improved spatial resolution of a system can decease the accuracy of assessment of relative myocardial perfusion, considering that the myocardium is moving with contraction. By including two versions of rotating Anger cameras, one with high resolution collimators and the other with focusing collimators, the investigators addressed the effects of improved spatial resolution via the astigmatic collimators on abrupt patient motion, although not directly on the effects of normal cardiac motion. Fortunately, even if it proves to be the case that improved spatial resolution exacerbates assessment of relative myocardial perfusion, methods have been established to correct for normal cardiac motion on perfusion assessment by repositioning cardiac counts to a common enddiastolic set of 3D coordinates. These techniques have been referred to as "motion frozen" cardiac corrections. $^{11}$

There are several virtues of the simulations reported by Salvadori et al., including use not only of unusually high resolution astigmatic collimators, and the recognition that different reconstruction algorithms and their image filtering options significantly affect the final system spatial resolution. Discrete displacements of a physical cardiac phantom in the 3 orthogonal directions were performed in a set of carefully controlled experiments. " $z$ " motion artifacts are most easily detected visually and by automated algorithms. ${ }^{12}$ Twisting motions of the patient, a component of " $y$ "' translations, are more challenging to detect, but nonetheless are important to recognize, because such patient motion certainly can adversely affect the analysis of relative myocardial perfusion. ${ }^{13}$ As potential sources of imaging artifacts in SPECT data by stationary SPECT systems have only recently started to be investigated, ${ }^{6}$ it is a welcome addition to have this added information regarding perfusion artifacts indexed to specific displacement amounts in specific directions.

There are some aspects of the investigation that might have been improved. More realistic phantom simulations would have been welcome, as those that were used had no attenuating medium. Radioactive sources in air have a much better spatial resolution and considerably less scatter of radiation into lower energy windows than those in attenuating media, so that the images used for this study may have been of an unrealistically too high a spatial resolution. This could have been improved through the use of torso phantoms that include simulated lung and spine inserts, and that use water in the intervening spaces to simulate tissue attenuation, but these were not used in this investigation. While it is understandable that eliminating attenuation as a variable is a sound approach in the course of conducting this experiment, it is well documented that attenuation artifacts can adversely seriously affect myocardial perfusion assessment. ${ }^{14}$ As such, it is important to note that almost all laboratories that employ attenuation corrections of myocardial perfusion SPECT studies do so by means of an accompanying CT scan as part of SPECT/CT systems that use rotating conventional Anger detectors. Non-rotating CZT systems that include $\mathrm{CT}$ attenuation correction capabilities are exceedingly rare. To mitigate attenuation problems for CZT SPECT systems, many laboratories have adopted prone imaging, and recognizing the limitations of doing so, some clinicians incorporate information obtained by CZT prone imaging along with supine images acquired by attenuation-corrected conventional rotating SPECT/ CT systems. ${ }^{15}$ The simulations performed by Salvadori et al. simulated patients in a supine rather than prone position. Consequently, the advantages of improved spatial resolution on motion artifacts that are suggested in this report must be weighed against the fact that hardly any systems currently in use also enable attenuation correction, which for many patients will be a far more troublesome complication. Of course, nuclear cardiologists whose imaging equipment does not enable 
attenuation corrections have needed to account for attenuation artifacts for many years, and those without CT components of SPECT/CT systems continue to do so, which is one reason that it would have been a welcome study design feature if the investigators had included visual assessments by nuclear cardiologists about the likelihood that the motion artifacts simulated in this report would actually have influenced or significantly altered their final clinical impressions of the presence and severity of cardiac disease. To have quantified analyses exclusively does have its place in experimental investigations of this sort, but ultimately quantified parameters are only one part of all of the information that a nuclear cardiologist integrates into forming the final impression, and visual analysis is generally the dominant determining factor.

Also, realistic torso phantoms have the further advantage of affording a set distance of the simulated anterior myocardium and of the anterior portion of the thorax to the camera detectors for rotating SPECT cameras. There was not adequate information provided in this report by Salvadori to be sure that the distances of simulated myocradia to detectors was realistic for the rotating SPECT systems, and as mentioned above, the CZT systems had the phantoms positioned as if for supine imaging rather than the prone positioning that is actually used for patients imaged with CZT devices. Spatial resolution worsens as distance from sources to detectors increase for all collimators, including the astigmatic and parallel-hole collimators of the rotating SPECT systems and the pinhole collimators of the CZT system used for this report. ${ }^{16}$ Distance to the detectors is an important variable, because the radioactive sources may have been too close to the CZT detectors, and too far from the rotating Anger detectors, to produce realistic simulations. Without more information, it is difficult to know whether or not the spatial resolution advantages of the CZT systems over the two rotating SPECT systems may be realistic or may have been unduly exaggerated.

In summary, it is certainly a worthwhile endeavor to test imaging systems with known quantities of isotopes in carefully constructed phantom experiments to facilitate comparisons among different devices of numerous imaging characteristics, including the effects of specific types of patient motion. The extent to which clinicians can directly apply the results reported for any particular set of phantom simulation experiments will depend strongly on the degree to which those experiments match the details of an individual laboratory's acquisition and processing protocols, including the manner in which quantitative measures are synthesized with visual impressions in the formation of the final diagnosis for a given patient. Nuclear cardiologists wishing to determine whether they can be guided by the results of simulations must decide whether those simulations, acquisition parameters, reconstruction choices and reporting systems adequately reproduce their own practices.

\section{Disclosure}

Kenneth Nichols participates in royalties from Syntermed, Inc., in relation to some of the algorithms discussed in this manuscript. Andrew Van Tosh serves as a consultant to Astellas Pharmaceuticals, Inc.

\section{References}

1. Prigent F, Maddahi J, Garcia E, Van Train K, Friedman J, Berman D. Noninvasive quantification of the extent of jeopardized myocardium in patients with single-vessel coronary disease by stress thallium-201 single-photon emission computerized rotational tomography. Am Heart J 1986;111:578-86.

2. Friedman J, Berman DS, Van Train K, Garcia EV, Bietendorf J, Prigent $\mathrm{F}$, et al. Patient motion in thallium-201 myocardial SPECT imaging. An easily identified frequent source of artifactual defect. Clin Nucl Med 1988;13:321-4.

3. Cooper JA, Neumann PH, McCandless BK. Effect of patient motion on tomographic myocardial perfusion imaging. J Nucl Med 1992;33:1566-71

4. Salvadori J, Petegnief Y, Sabbah R, Morel O, Boulahdour H, Karcher G, et al. Compared vulnerabilities to small cardiac motions between different cameras used for myocardial perfusion imaging. J Nucl Cardiol 2018 (current journal issue).

5. Slomka PJ, Patton JA, Berman DS, Germano G. Advances in technical aspects of myocardial perfusion SPECT imaging. J Nucl Cardiol 2009;16:255-76.

6. Redgate S, Barber DC, Fenner JW, Al-Mohammad A, Taylor JC, Hanney MB, et al. A study to quantify the effect of patient motion and develop methods to detect and correct for motion during myocardial perfusion imaging on a CZT solid-state dedicated cardiac camera. J Nucl Cardiol 2016;23:514-26.

7. Koshino K, Watabe H, Hasegawa S, Hayashi T, Hatazawa J, Iida $\mathrm{H}$. Development of motion correction technique for cardiac ${ }^{15} \mathrm{O}-$ water PET study using an optical motion tracking system. Ann Nucl Med 2010;24:1-11.

8. Dawood M, Gigengack F, Jiang X, Schäfers KP. A mass conservation-based optical flow method for cardiac motion correction in 3D-PET. Med Phys 2014;40(1):012505.

9. Tang J, Wang X, Gao X, Paul Segars W, Lodge MA, Rahmim A. Enhancing ejection fraction measurement through $4 \mathrm{D}$ respiratory motion compensation in cardiac PET imaging. Phys Med Biol 2017;62:4496-513.

10. Nichols KJ, Galt JR. Quality control for SPECT imaging. In: DePuey EG, Berman DS, Garcia EV, editors. Cardiac SPECT. 2nd ed. Philadelphia: Lippincott Williams \& Wilkins; 2001. p. 17-39.

11. Slomka PJ, Nishina H, Berman DS, Kang X, Akincioglu C, Friedman JD, et al. "Motion-frozen"' display and quantification of myocardial perfusion. J Nucl Med 2004;45:1128-34.

12. Matsumoto N, Berman DS, Kavanagh PB, Gerlach J, Hayes SW, Lewin HC, et al. Quantitative assessment of motion artifacts and validation of a new motion correction program for myocardial perfusion SPECT. J Nucl Med 2001;42:687-94. 
13. Agarwal V, DePuey EG. Myocardial perfusion SPECT horizontal motion Artifact. J Nucl Cardiol 2014;21:1260-5.

14. Singh B, Bateman TM, Case JA, Heller G. Attenuation artifact, attenuation correction, and the future of myocardial perfusion SPECT. J Nucl Cardiol 2007;14:153-64.

15. Ito S, Endo A, Okada T, Nakamura T, Sugamori T, Takahashi N, et al. Comparison of CTAC and prone imaging for the detection of coronary artery disease using CZT SPECT. Ann Nucl Med 2017;31:629-35.

16. Cherry SR. The gamma camera: performance characteristics. In: Cherry SR, Sorensen JA, Phelps ME, editors. Physics in nuclear medicine. 4th ed. Philadelphia: Saunders Elsevier; 2012. p. 20932. 